ORIGINAL ARTICLE

\title{
Pregnancy and contraceptive use among women participating in an HIV prevention trial in Tanzania
}

\author{
Aderonke Odutola, ${ }^{1}$ Kathy Baisley, ${ }^{1}$ Richard J Hayes, ${ }^{1}$ Mary Rusizoka, ${ }^{2}$ Clare Tanton, ${ }^{1}$ \\ Helen A Weiss, ${ }^{1}$ John Changalucha, ${ }^{3}$ David A Ross, ${ }^{1}$ Deborah Watson-Jones ${ }^{1,2,4}$
}

\begin{abstract}
- Additional figures are published online only. To view these files please visit the journal online (http://dx.doi.org/ 10.1136/sextrans-2011050412).

${ }^{1}$ London School of Hygiene and Tropical Medicine, London, UK ${ }^{2}$ The African Medical and Research Foundation, Mwanza, Tanzania

${ }^{3}$ The National Institute for Medical Research, Tanzania, Mwanza, Tanzania

${ }^{4}$ Mwanza Intervention Trials Unit, National Institute for Medical Research, Tanzania, Mwanza, Tanzania
\end{abstract}

\section{Correspondence to} Dr Deborah Watson-Jones, Faculty of Infectious and Tropical Diseases, Keppel St., London WC1E 7HT, UK; deborah.watson-jones@Ishtm. ac.uk

Accepted 26 February 2012

Published Online First 21 March 2012

\section{ABSTRACT}

Objectives Information on pregnancy rates and factors associated with pregnancy and contraceptive use is important for clinical trials in women in sub-Saharan Africa where withdrawal of investigational products may be required in the event of pregnancy with a consequent effect on sample size and trial power.

Methods A prospective cohort analysis of pregnancy and contraceptive use was conducted in Tanzanian women enrolled in a randomised placebo-controlled trial of herpes simplex virus-suppressive therapy with acyclovir to measure the effect on HIV incidence in HIVnegative women and on genital and plasma HIV viral load in HIV-positive women. The cohort was followed every 3 months for 12-30 months. Women at each visit were categorised into users or non-users of contraception. Pregnancy rates and factors associated with pregnancy incidence and contraceptive use were measured.

Results Overall 254 of 1305 enrolled women became pregnant at least once during follow-up (pregnancy rate: 12.0/100 person-years). Younger age, being unmarried, higher baseline parity and changes in contraceptive method during follow-up were independently associated with pregnancy. Having paid sex and being HIV positive were associated with lower risk of pregnancy. Uptake of contraception was associated with young age, being unmarried, occupation, parity and the number and type of sexual partners.

Conclusions Data on use of contraceptive methods and risk factors for pregnancy can help to guide decisions on trial eligibility and the need for additional counselling. Mandatory reliable contraceptive use in study participants may be required to reduce pregnancy rates in studies where pregnancy is contraindicated.

\section{INTRODUCTION}

HIV/AIDS continues to be a disease of major public health importance. ${ }^{1}$ In sub-Saharan Africa, HIV incidence is particularly high among women engaged in occupations where they may receive payment for sex in order to supplement their income..$^{2-4}$ A number of large-scale randomised controlled trials evaluating the safety and effectiveness of new HIV prevention biological interventions have recently been completed. ${ }^{5} 6$ The target population for many of these trials are HIVuninfected women at high risk of HIV in subSaharan Africa. However, the same group of women targeted for these trials also have high rates of pregnancy, even those who state at enrolment that they do not intend to become pregnant for the trial duration..$^{7-10}$ Pregnancy is contraindicated for many new biomedical interventions due to concerns about fetal safety. Participants who become pregnant may need to be withdrawn from the trial, reducing the statistical power to detect an impact of the intervention. ${ }^{10}$ It is therefore important to identify risk factors for pregnancy in specific populations of potential trial participants to help decide eligibility criteria and to develop pregnancy prevention strategies for future trials. ${ }^{11}$

We investigated the pregnancy rate, risk factors for pregnancy and determinants of contraceptive use in a randomised controlled trials of herpes simplex virus (HSV)-suppressive therapy for HIV prevention in north-western Tanzania.

\section{METHODS}

We undertook a cohort analysis of women who took part in a randomised placebo-controlled trial of the effects of HSV-suppressive therapy with acyclovir $400 \mathrm{mg}$ twice daily on HIV incidence in initially HIV-negative women and on genital and plasma HIV viral load in HIV-positive women. Full details of this trial have been published previously. $^{12}{ }^{13}$ Briefly, women aged $16-35$ years in 19 communities in north-western Tanzania who worked in bars and food and hospitality venues were enrolled between January 2004 and May 2006. At screening, blood was taken for HSV-2 and HIV serology. At enrolment, inclusion criteria were being HSV-2 seropositive, understanding the study aims, not planning to move in the next 24 months, a negative pregnancy test, not planning to become pregnant within the next 24 months, not breast feeding, no history of epilepsy and willing to attend mobile clinics. Eligible participants were randomised to receive acyclovir $400 \mathrm{mg}$ twice daily or placebo and were followed up every 3 months for 12-30 months. Women were enrolled in three phases: phases 1 and 2 were followed up for 30 months and phase 3 for 12 months. The intervention showed no significant effect on the HIV-related outcomes.

During each visit, participants were asked about their sexual behaviour since the last visit, symptoms of sexually transmitted infections and were counselled about risk reduction and family planning. Women were asked if they thought they might be pregnant, what method of contraception they were currently using and, if they were not receiving contraceptives through another service, were offered the following contraceptives for free: combined oral contraceptive (COC), injectable mi.com/site/about/unlocked. xhtm 
depot medroxyprogesterone acetate (Depo-provera) or condoms. At each visit, the participant was tested for pregnancy if her last menstrual period (LMP) was late or if she thought she might be pregnant. Routine pregnancy testing was not done because women were only seen every 3 months and testing women whose LMP was more than 4 weeks ago should have detected most pregnancies and limited the detection of non-viable chemical pregnancies. Further trial tablets were issued unless the participant was pregnant or had to stop taking trial tablets for other reasons in which case participants stayed in follow-up unless they specifically asked to withdraw. Voluntary HIV counselling and testing was offered at every visit.

Ethical approval for the trial was given by the ethics committees of the Tanzanian Medical Research Coordinating Committee and the London School of Hygiene and Tropical Medicine (Current Controlled Trials number ISRCTN35385041).

This study is a secondary analysis of trial data that were analysed using Stata V.10.1 (College Station). Pregnancy rates were calculated as number of pregnancies/person-years of follow-up. Women were censored at the earliest of date of first pregnancy, date last seen or end of study. Women who became pregnant more than once contributed person-years only to the time of their first pregnancy. Since pregnancy tests were not done at every visit, it was assumed that the subject was not pregnant if a test was not performed. Date of conception was calculated as midway between the date of first positive pregnancy test and the previous visit date, if this period was $\leq 9$ months. If the period was over 9 months, then date of conception was assumed to be 4.5 months before the date of first positive pregnancy test. To check the sensitivity of the results to the definition of date of conception, two additional methods were used to estimate the conception date. First, the conception date was estimated as 40 weeks before the delivery date, if that information was available (no pre-term births were recorded in the trial). Second, the conception date was estimated as midway between the LMP date of and first positive pregnancy test.

Poisson regression was used to estimate RR for the association of pregnancy with baseline factors and time-varying exposures measured during follow-up and trends in pregnancy rates over trial follow-up.

Contraceptive use at each visit was categorised as any modern method (hormonal methods, the intra-uterine contraceptive device (IUD) or condoms) or other method. ORs for factors associated with modern contraception use during follow-up were estimated using random effects logistic regression to account for within-woman correlations. The enrolment visit was excluded from the analysis. Trends over time in the use of hormonal methods and condoms were assessed, restricted to women who attended the 30-month visit. Condom use was defined from responses to the question "what method are you currently using for family planning?' and did not include condom use for other purposes.

Women who were surgically sterile at enrolment were excluded from the pregnancy risk factor analyses (as they were not at risk of pregnancy) and contraceptive use. They were included in the calculation of pregnancy rates as they contributed to the person-years of follow-up.

A conceptual framework was used to build a multivariable model for factors associated with pregnancy and with modern contraception. ${ }^{14}$ Age was considered an a priori confounder. Baseline socio-demographic factors whose age-adjusted association reached $\mathrm{p}<0.10$ were included in an initial multivariable model and retained if they remained independently associated with the outcome at $p<0.10$. Baseline behavioural factors were added to this model one by one, and those remaining significant at $p<0.10$ were retained. Associations with time-varying behavioural factors were determined in a similar way. The final model excluded factors one at a time, starting with the factor with the largest $p$ value, until all remaining factors were significant at $\mathrm{p}<0.05$.

\section{RESULTS}

Socio-demographic and behavioural characteristics at baseline Overall 1305 women were enrolled, 652 (50.0\%) were randomised to acyclovir and 653 to placebo. The mean (SD) age of participants at screening was 27.6 (4.9) years. Over half the women (54.3\%) were divorced, separated or widowed and $85.7 \%$ had at least one child (table 1). Over half of those enrolled (54.9\%) stated that they wanted to have children in the future

At enrolment, $54.0 \%$ were not using any form of contraception and $29.6 \%$ were using hormonal contraceptives (table 1 ). Overall, 37.1\% women were HIV positive. Over half $(60.3 \%)$ accepted Voluntary HIV counselling and testing and were informed of their HIV status at enrolment, including $56.4 \%$ of HIV-positive women.

\section{Pregnancy rates and outcomes}

During the trial, 254 (19.5\%) women became pregnant at least once, giving a pregnancy rate of $12.0 / 100$ person-years $(95 \% \mathrm{CI}$ 10.6 to 13.6/100 person-years; table 2), and 22 women became pregnant twice. Pregnancy rates were similar among phases 1 and 2 women enrolled for 30 months (12.0, 95\% CI 10.5 to 13.7) and phase 3 women enrolled for 12 months (12.2, 95\% CI 8.5 to 17.5 ; table 2). Pregnancy rates were highest in the first 3 months of the trial with weak evidence of a decreasing trend in pregnancy rates over the study duration (RR for linear trend from 1 year to the next $=0.85 /$ year, $95 \%$ CI 0.72 to $1.01, p=0.07$; supplemental figure 1). HIV-uninfected women had higher pregnancy rates, particularly in younger age groups (supplemental figure 1). Pregnancy rates were similar using the two alternative methods of estimating the conception date (11.4/100 person-years, $95 \% \mathrm{CI}$ 10.0 to 12.9 based on delivery dates; $11.8 / 100$ person-years, $95 \%$ CI 10.4 to 13.4 based on LMP). Of the 276 pregnancies, 163 $(59.1 \%)$ were full-term live births (table 2$)$. The outcomes of 74 (26.8\%) pregnancies were not known.

\section{Risk factors for pregnancy}

In univariate analysis, factors associated with incident pregnancy included younger age, lower education level, being unmarried, occupation, contraceptive method in the preceding 3 months, changing contraceptive methods between visits, fewer sexual partners in the past 3 months, not having paid sex and being HIV uninfected (table 3). Among contraceptive users, the lowest pregnancy rates were in those using injectable hormonal contraception (3.68/100 person-years). Pregnancy rates were similar for condom and COC users (10.21/100 personyears and 10.77/100 person-years, respectively).

In multivariate analysis, younger age, being single (adjusted RR (aRR) 1.76, 95\% CI 1.18 to 2.64), higher parity and not using contraception (aRR 5.84, 95\% CI 4.31 to 7.91) were independently associated with pregnancy (table 3 ). Having paid sex in the last 3 months (aRR 0.59, 95\% CI 0.43 to 0.81), using injectable contraception and being HIV positive (aRR $0.55,95 \%$ CI 0.42 to 0.73 ) were inversely associated with pregnancy. Number of children and age were strongly confounded, and the effect of each of these variables on pregnancy rate was strengthened after adjusting for the other variable. 
Table 1 Baseline socio-demographic and behavioural characteristics of the women in the cohort

\begin{tabular}{|c|c|}
\hline & $\mathbf{N}(\%)$ \\
\hline \multicolumn{2}{|l|}{ Socio-demographic characteristics } \\
\hline \multicolumn{2}{|l|}{ Age (years) } \\
\hline $16-19$ & $71(5.4)$ \\
\hline $20-24$ & $289(22.2)$ \\
\hline $25-29$ & $424(32.5)$ \\
\hline $30-35$ & $521(39.9)$ \\
\hline \multicolumn{2}{|l|}{ Educational level } \\
\hline None & $153(11.7)$ \\
\hline Incomplete primary & $228(17.5)$ \\
\hline Completed primary or higher & $924(70.8)$ \\
\hline \multicolumn{2}{|l|}{ Marital status } \\
\hline Married/living as married & $328(25.1)$ \\
\hline Divorced/separated/widowed & $708(54.3)$ \\
\hline Single & $269(20.6)$ \\
\hline \multicolumn{2}{|l|}{ Occupational group at screening } \\
\hline Local food handler & $661(50.7)$ \\
\hline Guesthouse & $139(10.7)$ \\
\hline Bar worker & $208(15.9)$ \\
\hline Local brew seller & $95(7.3)$ \\
\hline Restaurant/cafe/grocery & $202(15.5)$ \\
\hline \multicolumn{2}{|l|}{ Number of children ever } \\
\hline None & $186(14.3)$ \\
\hline 1 & $337(25.8)$ \\
\hline $2-4$ & $679(52.0)$ \\
\hline$\geq 5$ & $103(7.9)$ \\
\hline \multicolumn{2}{|l|}{ Wish to have children in future } \\
\hline No & $572(44.0)$ \\
\hline Yes & $714(54.9)$ \\
\hline Don't know & $15(1.1)$ \\
\hline \multicolumn{2}{|l|}{ Behavioural characteristics } \\
\hline \multicolumn{2}{|l|}{ Any regular partner } \\
\hline Yes & $1084(83.1 \%)$ \\
\hline No & $221(16.9 \%$ \\
\hline \multicolumn{2}{|l|}{ Living with partner* } \\
\hline In the same house & $343(26.5)$ \\
\hline Apart, meet weekly to $<3$ monthly & $521(40.2)$ \\
\hline Apart, meet $>3$ monthly & $210(16.2)$ \\
\hline No regular partner & $221(17.1)$ \\
\hline \multicolumn{2}{|l|}{ Number of sexual partners in the last $3 \mathrm{~m}$} \\
\hline $0-1$ & 769 (58.9) \\
\hline 2 & $325(24.9)$ \\
\hline$\geq 3$ & 43 (16.2) \\
\hline \multicolumn{2}{|l|}{ Paid sex in the past $3 \mathrm{~m}$} \\
\hline No & $806(61.8)$ \\
\hline Yes & 499 (38.2) \\
\hline \multicolumn{2}{|l|}{ Contraceptive use at enrolment } \\
\hline None & $705(54.0)$ \\
\hline Combined oral contraceptive & $144(11.0)$ \\
\hline Injection & $243(18.6)$ \\
\hline Condom & $91(7.0)$ \\
\hline Rhythm/withdrawal/traditional & $74(5.7)$ \\
\hline Intrauterine contraceptive device & $6(0.5)$ \\
\hline Surgical sterilisation & $42(3.2)$ \\
\hline \multicolumn{2}{|l|}{ HIV status at enrolment } \\
\hline Negative & $821(62.9)$ \\
\hline Positive & $484(37.1)$ \\
\hline
\end{tabular}

*65 missing values.

The highest rate of pregnancy was during periods when women stopped using modern contraception (100.5/100 personyears). After adjusting for other factors, when women stopped using modern contraception, they were at 11 times the risk of
Table 2 Pregnancy incidence and outcomes

\begin{tabular}{ll}
\hline Total number of pregnancies & 276 \\
Number of women with 1 pregnancy & 232 \\
Number of women with $>1$ pregnancy & 22 \\
Median (IOR) time from enrolment to first pregnancy in years* & \\
All women & $0.85(0.35-1.56)$ \\
Women enrolled in phases 1 and 2 (30-month follow-up) & $1.04(0.36-1.60)$ \\
Women enrolled in phase 3 (12-month follow-up) & $0.42(0.14-0.68)$ \\
Pregnancy incidence during follow-up (per 100 person-years) † & \\
All women & $12.0(10.6-13.6)$ \\
Women enrolled in phases 1 and 2 (30-month follow-up) & $12.0(10.5-13.7)$ \\
Women enrolled in phase 3 (12-month follow-up) & $12.2(8.5-17.5)$ \\
Pregnancy outcome & \\
Term live birth & $163(59.1 \%)$ \\
Premature live birth & $0(-)$ \\
Stillbirth & $4(1.4 \%)$ \\
Miscarriage & $24(8.7 \%)$ \\
Termination & $10(3.6 \%)$ \\
Ectopic pregnancy & $1(0.4 \%)$ \\
Unknown & $74(26.8 \%)$ \\
Total & $276(100 \%)$ \\
\hline *Among 254 women who became pregnant at least once. & \\
†lncidence restricted to first pregnancy. &
\end{tabular}

pregnancy than when women used modern contraception without changing method, while during periods when women used no or non-modern contraception, they were at about four times the risk of pregnancy (table 3 ). In contrast, when women changed between modern contraceptive methods, they were at lower risk of pregnancy than during periods when there was no change ( $\mathrm{aRR}=0.32,95 \% \mathrm{CI} 0.13$ to 0.77$)$. Of the five pregnancies that occurred in women changing contraceptive methods, three became pregnant changing from hormonal methods to condoms, one when changing from condoms to a hormonal method and one when changing from injections to COC.

\section{Trends in contraceptive use}

Trends in contraceptive use were analysed in 520 women who completed the study at 30 months without becoming pregnant and who were not surgically sterilised. There was an increase in COC use from $11.0 \%$ at enrolment to $28.5 \%$ at 3 months, and this remained around $37 \%$ after 9 months (supplemental figure 2). Injection use increased from $20.6 \%$ at enrolment to $32.2 \%$ at 9 months but fell slightly thereafter and remained around $26 \%$. Similarly, condom use as a contraceptive method increased from $6.5 \%$ at enrolment to $23.6 \%$ at 3 months, with little change thereafter.

\section{Factors associated with modern contraceptive use during follow-up}

Young age, being unmarried, occupation at screening, religion, high parity, living apart from her partner, increasing sexual partners in the past 3 months, paid sex in the past 3 months and knowledge of positive HIV status were associated with modern contraceptive use on univariate analysis (table 4). On multivariate analysis, factors independently associated with modern contraception included younger age, being unmarried (adjusted OR $7.80,95 \%$ CI 2.83 to 21.52 for single compared with married), having a regular partner (adjusted OR 2.47, 95\% CI 1.19 to 5.13), occupation (highest among bar workers, lowest among local brew sellers), high parity and increasing sexual partners in the last 3 months (table 4). Women who were HIV positive and aware of their status were less likely to use modern contraception compared with HIV-positive women who were 
Table 3 Association between pregnancy and socio-demographic and behavioural factors

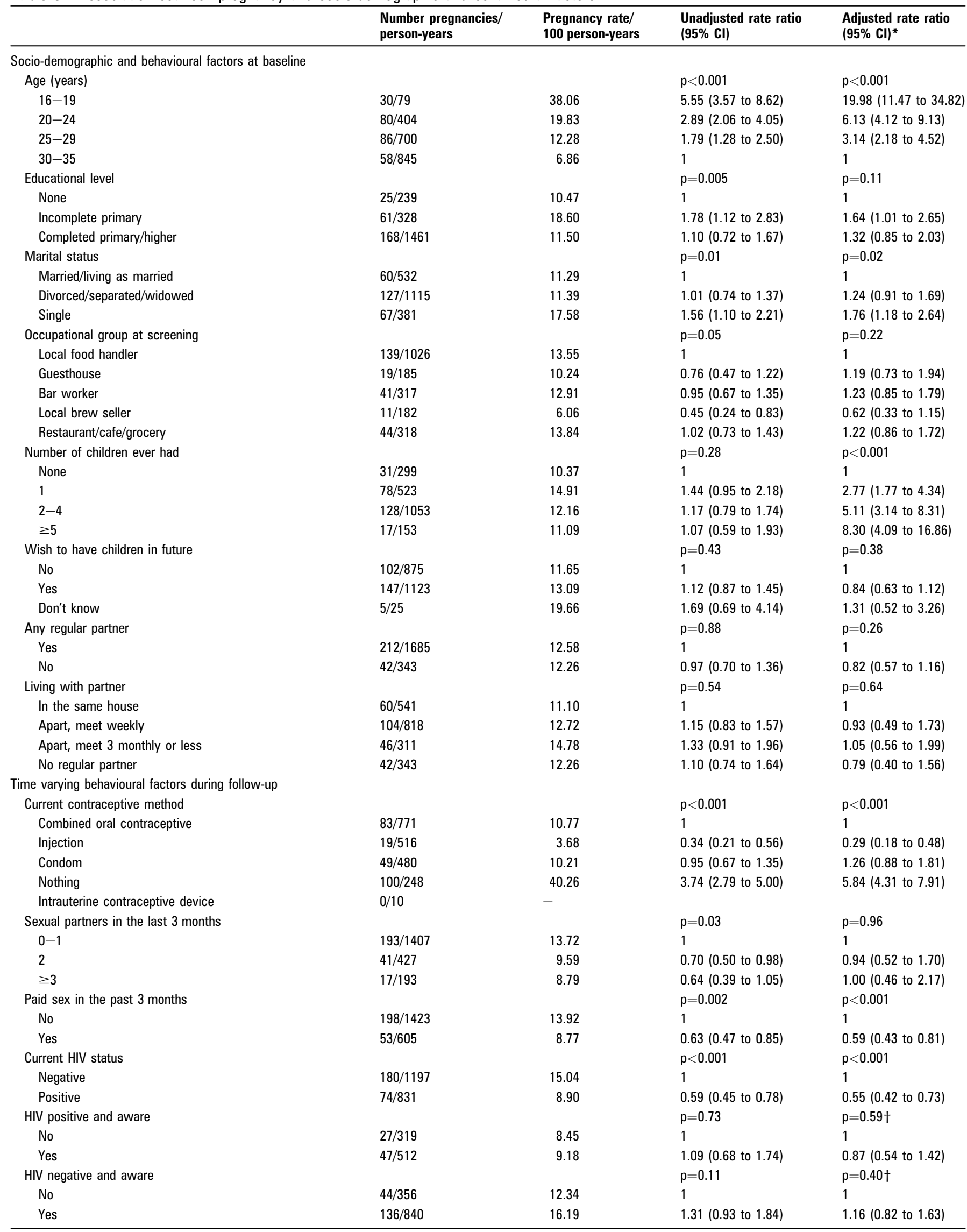


Table 3 Continued

\begin{tabular}{lllll}
\hline & $\begin{array}{l}\text { Number pregnancies/ } \\
\text { person-years }\end{array}$ & $\begin{array}{l}\text { Pregnancy rate/ } \\
\mathbf{1 0 0} \text { person-years }\end{array}$ & $\begin{array}{l}\text { Unadjusted rate ratio } \\
\text { (95\% Cl) }\end{array}$ & $\begin{array}{l}\text { Adjusted rate ratio } \\
\text { (95\% Cl)* }\end{array}$ \\
\hline $\begin{array}{l}\text { Change in contraceptive method } \neq \\
\quad \text { No change in method (using modern contraception) }\end{array}$ & $136 / 1417$ & & $\begin{array}{l}\mathrm{p}<0.001 \\
\mathbf{p}<0.001 \S\end{array}$ & 1 \\
$\quad$ Start modern contraception & $10 / 198$ & 9.60 & 1 & $0.56(0.29$ to 1.07$)$ \\
Stop modern contraception & $60 / 60$ & 5.04 & $0.53(0.28$ to 1.00$)$ & $11.16(8.18$ to 15.23$)$ \\
No change in method (not using modern contraception) & $40 / 188$ & 100.5 & $10.47(7.72$ to 14.18$)$ & $3.67(2.54$ to 5.29$)$ \\
Using modern contraception, change method & $5 / 162$ & 21.23 & $2.21(1.55$ to 3.15$)$ & $0.32(0.13$ to 0.77$)$ \\
\hline
\end{tabular}

*Adjusted for independent predictors of pregnancy: age group, marital status, number of children, current contraceptive method, paid sex in the past 3 months and HIV status.

†Both variables relating to HIV status and knowledge of HIV status included in model at the same time, adjusted for independent predictors of pregnancy listed in first footnote. Shows association between pregnancy rate and knowledge of HIV status separately among HIV-positive and HIV-negative women.

¥Contraceptive method reported at current visit compared with that reported at previous visit. Modern contraception defined as combined oral contraceptive (COC), injection, condom or IUCD. Analysed as a time-updated variable, so distinguishes between periods when women report no change in a particular method (eg, report using COC at current and previous visit), start/stop using a modern method or change between two modern methods (eg, using COC at current and condom at previous visit).

$\S$ Adjusted for all independent predictors of pregnancy listed in first footnote, except for current contraceptive method.

not aware of their status (adjusted $\mathrm{OR}=0.43,95 \%$ CI 0.21 to 0.87 ).

\section{DISCUSSION}

We found an overall pregnancy rate of 12.0/100 person-years (95\% CI 10.6 to 13.6$)$ in HIV prevention trial participants in Tanzania. HIV-negative women were at increased risk of pregnancy (rate $=15.0 / 100$ person-years). The uptake of modern contraceptives increased following enrolment.

In biomedical HIV prevention trials, women who become pregnant during the trial are generally withdrawn from investigational product use due to fetal safety concerns and their observations are censored, as was done in our study. Our results confirm those of other HIV prevention trials where a high rate of pregnancy is consistently reported despite offering free contraception services. $^{89} 111516$ Rates in these trials have varied from around $8 / 100$ to $52 / 100$ person-years. ${ }^{9} 1516$ This variation may be due to differences in fertility, the frequency of pregnancy testing or sex unprotected by contraceptive methods, availability and efficacy of contraception used and differences in adherence. We did not test for pregnancy routinely at every visit unless there was a suspicion of pregnancy, which may have missed some pregnancy losses. Conversely frequent pregnancy testing will lead to an increase in recorded pregnancies because this will identify chemical pregnancies, which do not become true pregnancies, and some early pregnancies, which would have been spontaneously or medically aborted. In one multicentre trial where pregnancy testing was conducted at quarterly intervals, $25.9 \%$ of the 228 positive pregnancy tests were negative at the next testing, presumably due to spontaneous or elective abortions. ${ }^{8}$

The factors associated with pregnancy in our study are similar to several previous studies. ${ }^{7} 1617$ Young Tanzanian women are who are fertile may avoid the use of hormonal contraception if they perceive that it could reduce their future chances of conceiving, as supported by reported preference for using condoms in young women. ${ }^{18} 19$ Multiparous women were also at higher risk of pregnancy, possibly because those who already have children may be more fertile or may desire to have more.

HIV-uninfected women had higher pregnancy rates than HIVinfected women, as shown in other studies. ${ }^{20-22}$ HIV infection has been associated with reduced fertility. ${ }^{20} 21$ HIV-infected women may also be conscious of their health needs and the potential burden of caring for a child and thus may be more cautious about becoming pregnant, ${ }^{23} 24$ although perceived high risk for HIV was associated with an increased desire for future pregnancies in Zimbabwe. ${ }^{25}$ In an HIV prevention trial in serodiscordant couples, pregnancy rates were similar in HIVinfected and HIV-uninfected women. ${ }^{26}$
Women who received payment for sex had lower pregnancy rates. This may be related to ability to negotiate condom use ${ }^{19}$ or to higher rates of sexually transmitted infections and their sequelae, such as pelvic inflammatory disease. ${ }^{27}$ Those with occasional paying partners may also have fewer total sex acts than women in regular partnerships.

Just over half of women in this trial were not using any form of contraceptive at enrolment, similar to studies in Madagascar and South Africa. ${ }^{1628}$ Uptake of condoms for contraception and hormonal contraception increased as soon as women entered the trial and contraceptive usage remained fairly stable throughout the study which suggests that counselling and family planning provision had an immediate and sustained effect on contraceptive practices after study entry. Stopping contraception was, not unexpectedly, associated with a very high risk of pregnancy. Women who consistently did not use modern contraceptives were also at an increased, but lower, risk of pregnancy.

Changes in family planning methods have primarily been described for women pre- and post-trial enrolment where trialspecific family planning counselling can influence uptake of condoms for example. ${ }^{29}$ In our trial, $73 \%$ of women changed contraceptive method at least once during follow-up but, with the exception of stopping contraception, this was not associated with a higher pregnancy risk compared with periods of consistent contraceptive method use.

Study participants who were HIV positive and aware of their status were less likely to use contraception. This has important implications for the risk of transmitting the virus to their partners or children, even if these women are less likely to become pregnant ${ }^{30-32}$ and emphasises the importance of offering free and comprehensive family planning services as part of standard of care of HIV-infected women.

Women who engage in paid sex may be more likely to use contraception because they may have one-time relationships, even if they have regular partners, and becoming pregnant may reduce their income. As mentioned above, these women may be able to better negotiate condom use. ${ }^{19}$ Women with regular partners were more likely to use modern contraception than those without regular partners, possibly because the latter are having less frequent sex or no sex at all. Unlike studies in developed countries, ${ }^{33}$ having a higher number of sexual partners in the past 3 months was associated with using contraception, which may be related to frequency of sex.

A limitation of our study is that we did not routinely test for pregnancy at each visit, so we may have underestimated the pregnancy rate in this cohort. In addition, we did not gather data on dual contraception method use. Strengths of the study include a large sample size, with data on current contraceptive 
Table 4 Factors associated with use of modern* contraceptive method during follow-up

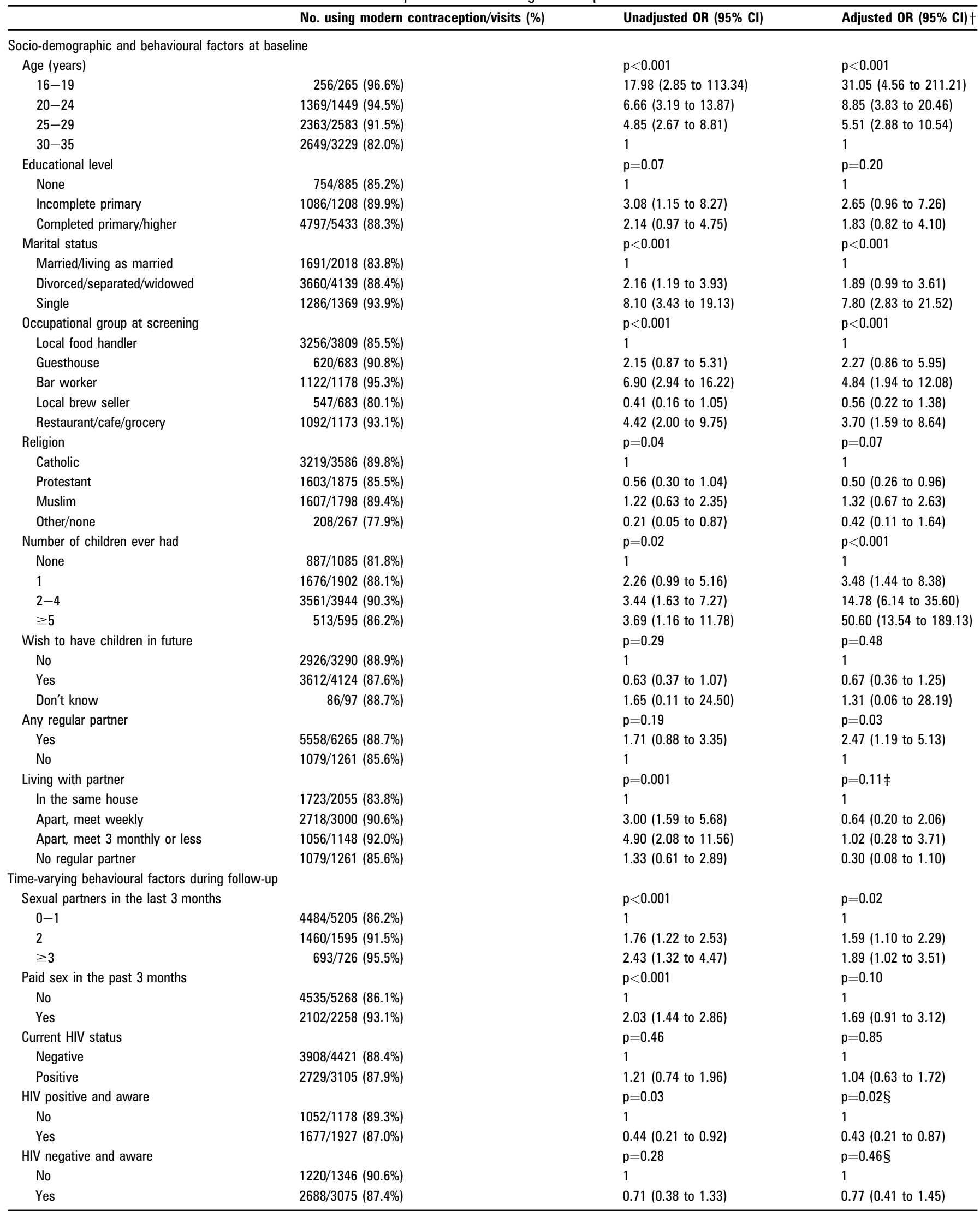

*Modern contraception defined as any hormonal method, IUCD or condoms.

†Adjusted for independent predictors of using contraception: age group, marital status, occupational group at screening, number of children, any regular partner and number of partners in the past 3 months.

$\ddagger$ Adjusted for all independent predictors of using contraception listed in second footnote, except for any regular partner.

$\S$ Both variables relating to HIV status and knowledge of HIV status included in the model at the same time, adjusted for independent predictors of using contraception listed in second footnote.

Shows association between using modern contraception and knowledge of HIV status separately among HIV-positive and HIV-negative women. 
Pregnancy in HIV prevention trials of biomedical interventions where the investigational product needs to be withdrawn impacts on trial power and generalisability of results.

- Pregnancy rates remain high in clinical trial participants in sub-Saharan Africa, even when contraception is provided free at trial clinics.

- Frequent pregnancy testing may detect chemical pregnancies that result in unnecessary withdrawal from trial product, while less frequent testing may miss early pregnancy losses.

- Receiving and using reliable contraception may need to become a mandatory eligibility criterion in future trials of biomedical interventions where pregnancy leads to withdrawal of trial product.

use gathered at 3-month intervals allowing us to look at the effect of changing contraceptive methods.

In conclusion, this study demonstrated a high incidence of pregnancy in an HIV prevention trial cohort and identified a number of factors that predict increased risk of pregnancy and use of effective modern contraceptives following study enrolment. Such information may be of value in selecting participants for future trials where pregnancy is contraindicated or identifying the need for more intensive counselling to explain that pregnancy may result in study withdrawal, in addition to family planning provision targeted to women who are at highest risk of pregnancy. Where it is essential to minimise pregnancy in study participants, mandatory use of an effective contraceptive method such as hormonal method or IUDs, alongside condoms for HIV prevention, would be the only approach likely to achieve this since self-reported pregnancy intentions are not reflected in actual pregnancy rates in many trials. However, recent findings have shown that hormonal contraception may increase the risk of HIV acquisition in HIV-negative women and female-to-male HIV transmission, although results from studies have not always been consistent and may depend on age and type of hormonal contraception. ${ }^{34-38}$ Further studies are urgently needed to determine which specific hormonal methods and which dose of hormone may be recommended in populations at risk of HIV. Until then, clear counselling about the potential risks and benefits of hormonal contraception, offering alternative contraceptive choices, such as IUD, and on-going condom promotion and provision will be essential when offering contraception for HIV prevention trial participants.

Acknowledgements We thank the Tanzanian Ministry of Health and Social Welfare and the Director General of the National Institute for Medical Research, Tanzania, for permission to conduct and publish the study. We are grateful to Geita Gold Mine for logistic support and to the administrative staff in AMREF, LSHTM and the NIMR/AMREF/LSHTM collaborative projects office. We thank all the women who participated in this study.

Contributors DW-J, RJH, DAR, CT, HW and JC conceived the study and prepared the protocol. DW-J obtained the funding and had overall responsibility for supervision and conduct of the study. MR supervised the field teams and data collection. Data analysis was done by $A 0$ and $K B$. $A 0$ prepared the first draft of the manuscript. All authors commented on and contributed to the final version of the manuscript.

Funding The Wellcome Trust, the United Kingdom Medical Research Council and the United Kingdom Department for International Development.

Competing interests None.

Ethics approval Ethics approval was provided by Medical Research Coordinating Committee of Tanzania and London School of Hygiene and Tropical Medicine ethics committee.

\section{REFERENCES}

1. UNAIDS. 2008 Report on the global AIDS epidemic. http://www.unaids.org/en/ dataanalysis/epidemiology/2008reportontheglobalaidsepidemic/ (accessed 10 Jun 2011).

2. Watson-Jones D, Weiss HA, Rusizoka M, et al. Risk factors for herpes simplex virus type 2 and HIV among women at high risk in northwestern Tanzania: preparing for an HSV-2 intervention trial. J Acquir Immune Defic Syndr 2007; 46:631-42.

3. Riedner G, Rusizoka M, Hoffmann 0, et al. Baseline survey of sexually transmitted infections in a cohort of female bar workers in Mbeya Region, Tanzania. Sex Transm Infect 2003;79:382-7.

4. Kapiga SH, Sam NE, Shao JF, et al. HIV-1 epidemic among female bar and hotel workers in northern Tanzania: risk factors and opportunities for prevention. J Acquir Immune Defic Syndr 2002;29:409-17.

5. Padian NS, Buve A, Balkus J, et al. Biomedical interventions to prevent HIV infection: evidence, challenges, and way forward. Lancet 2008;372:585-99.

6. Padian NS, McCoy SI, Karim SS, et al. HIV prevention transformed: the new prevention research agenda. Lancet 2011;378:269-78.

7. Halpern V, Lie CC, Feldblum P, et al. Predictors of pregnancy in microbicide trials. Contraception 2011:83:436-40.

8. Reid SE, Dai JY, Wang J, et al. Pregnancy, contraceptive use, and HIV acquisition in HPTN 039: relevance for HIV prevention trials among African women. J Acquir Immune Defic Syndr 2010;53:606-13.

9. Peterson L, Taylor D, Roddy R, et al. Tenofovir disoproxil fumarate for prevention of HIV infection in women: a phase 2, double-blind, randomized, placebo-controlled trial. PLoS Clin Trials 2007;2:e27.

10. Raymond EG, Taylor D, Cates W Jr, et al. Pregnancy in effectiveness trials of HIV prevention agents. Sex Transm Dis 2007;34:1035-9.

11. Ramjee G, Kapiga $S$, Weiss $S$, et al. The value of site preparedness studies for future implementation of phase 2/llb/III HIV prevention trials: experience from the HPTN 055 study. J Acquir Immune Defic Syndr 2008:47:93-100.

12. Watson-Jones D, Weiss HA, Rusizoka M, et al. Effect of herpes simplex suppression on incidence of HIV among women in Tanzania. N Engl J Med 2008;358:1560-71.

13. Tanton C, Weiss HA, Rusizoka M, et al. Long-term impact of acyclovir suppressive therapy on genital and plasma HIV RNA in Tanzanian women: a randomized controlled trial. J Infect Dis 2010;201:1285-97.

14. Victora CG, Huttly SR, Fuchs SC, et al. The role of conceptual frameworks in epidemiological analysis: a hierarchical approach. Int J Epidemiol 1997;26:224-7.

15. Skoler-Karpoff S, Ramjee G, Ahmed K, et al. Efficacy of Carraguard for prevention of HIV infection in women in South Africa: a randomised, double-blind, placebocontrolled trial. Lancet 2008;372:1977-87.

16. Feldblum PJ, Nasution MD, Hoke TH, et al. Pregnancy among sex workers participating in a condom intervention trial highlights the need for dual protection. Contraception 2007;76:105-10.

17. Forsyth AD, Coates TJ, Grinstead OA, et al. HIV infection and pregnancy status among adults attending voluntary counseling and testing in 2 developing countries. Am J Public Health 2002;92:1795-800.

18. Otoide V, Oronsaye F, Okonofua E. Why Nigerian adolescents seek abortion rathe than contraception: evidence from focus group discussion. Int Fam Plan Perspect 2001;3:77-81

19. Mgalla Z, Pool R. Sexual relationships, condom use and risk perception among female bar workers in north-west Tanzania. AIDS Care 1997;9:407-16.

20. Gray RH, Wawer MJ, Serwadda D, et al. Population-based study of fertility in women with HIV-1 infection in Uganda. Lancet 1998;351:98-103.

21. Zaba B, Gregson S. Measuring the impact of HIV on fertility in Africa. AIDS 1998;12 (Suppl 1):S41-50.

22. Kongnyuy EJ, Wiysonge CS. Association between fertility and HIV status: what implications for HIV estimates? BMC Public Health 2008;8:309.

23. Cooper D, Harries J, Myer L, et al. "Life is still going on": reproductive intentions among HIV-positive women and men in South Africa. Soc Sci Med 2007;65:274-83

24. Johnson KB, Akwara P, Rutstein SO, et al. Fertility preferences and the need for contraception among women living with HIV: the basis for a joint action agenda. AIDS 2009;23(Suppl 1):S7-17.

25. Moyo W, Mbizvo MT. Desire for a future pregnancy among women in Zimbabwe in relation to their self-perceived risk of HIV infection, child mortality, and spontaneous abortion. AIDS Behav 2004:8:9-15.

26. Ngure K, Heffron R, Mugo N, et al. Contraceptive method and pregnancy incidence among African women in HIV-1 serodiscordant relationships. AIDS 2012;26:513-18.

27. Ward H, Day S. What happens to women who sell sex? Report of a unique occupational cohort. Sex Transm Infect 2006;82:413-17.

28. Subramanian L, McGrath N, Ndlovu H, et al. Family planning methods among women in a vaginal microbicide feasibility study in rural KwaZulu-Natal, South Africa. Afr J Reprod Health 2008;12:45-63.

29. Macqueen KM, Johnson L, Alleman $P$, et al. Pregnancy prevention practices among women with multiple partners in an HIV prevention trial. J Acquir Immune Defic Syndr 2007;:46:32-8. 
30. Brou H, Viho I, Djohan G, et al. Contraceptive use and incidence of pregnancy among women after HIV testing in Abidjan, Ivory Coast. Rev Epidemiol Sante Publique 2009;57:77-86

31. Finocchario-Kessler S, Sweat MD, Dariotis JK, et al. Understanding high fertility desires and intentions among a sample of urban women living with HIV in the United States. AIDS Behav 2010:14:1106-14.

32. MacPhail C, Pettifor AE, Pascoe S, et al. Contraception use and pregnancy among 15-24 year old South African women: a nationally representative cross-sectional survey. BMC Med 2007:5:31.

33. Radcliffe KW, Tasker T, Evans BA, et al. A comparison of sexual behaviour and risk behaviour for HIV infection between women in three clinical settings. Genitourin Med 1993:69:441-5.
34. Baeten JM, Benki S, Chohan V, et al. Hormonal contraceptive use, herpes simplex virus infection, and risk of HIV-1 acquisition among Kenyan women. AIDS 2007:21:1771-7.

35. Watson-Jones D, Baisley K, Weiss HA, et al. Risk factors for HIV incidence in women participating in an HSV suppressive treatment trial in Tanzania. AIDS 2009:23:415-22

36. Morrison CS, Chen PL, Kwok C, et al. Hormonal contraception and HIV acquisition: reanalysis using marginal structural modeling. AIDS 2010;24:1778-81.

37. Morrison CS, Skoler-Karpoff S, Kwok C, et al. Hormonal contraception and the risk of HIV acquisition among women in South Africa. AIDS 2012;26:497-504.

38. Heffron R, Donnell D, Rees $\mathrm{H}$, et al. Use of hormonal contraceptives and risk of HIV-1 transmission: a prospective cohort study. Lancet Infect Dis 2012;12:19-26. 